
\title{
25 Research Square \\ E3 ubiquitin ligase FBW7-mediated ubiquitination of EZH2 prevents type I diabetes in NOD mice
}

Jiamei Tian

Department of Pediatrics, Linyi People's Hospital, Linyi 276000, P.R. China

\section{Yuanyuan Zhang}

Department of Endocrinology, Linyi People's Hospital, Linyi 276000, P.R. China

Shanshan Li ( $\sim$ lishanshan20200301@163.com )

Linyi People's Hospital https://orcid.org/0000-0003-0082-2971

\section{Research}

Keywords: FBW7, EZH2, ZBTB16, Type I diabetes, Ubiquitination, Methylation, Pancreatic beta cell

Posted Date: April 29th, 2020

DOl: https://doi.org/10.21203/rs.3.rs-24086/v1

License: (1) This work is licensed under a Creative Commons Attribution 4.0 International License. Read Full License 


\section{Abstract \\ Background}

Type I diabetes (T1D) is a heritable autoimmune disease targeting pancreatic beta cells. This study explores the molecular mechanism of E3 ubiquitin ligase FBW7 in reducing the occurrence of T1D.

\section{Methods}

The protein expression of FBW7, EZH2, and ZBTB16 was quantified in non-obese diabetic (NOD) mouse model of streptozotocin-induced T1D. Next, splenocytes and pancreatic beta cells were isolated to measure production of pro-inflammatory cytokines in splenocytes, as well as islet beta-cell apoptosis. Besides, the stability of EZH2 induced by FBW7 was analyzed by cycloheximide chase assay. The binding affinity of FBW7 and EZH2 and the consequence of ubiquitination were monitored by immunoprecipitation (IP) assay. Last, chromatin immunoprecipitation (ChIP) assay was employed to determine the accumulation of EZH2 and H3K27me3 at the ZBTB16 promoter region.

\section{Results}

We found downregulated FBW7 and ZBTB16, and upregulated EZH2 in diabetic NOD mice. Overexpression of FBW7 in the NOD mice inhibited the production of pro-inflammatory cytokines in the splenocytes and the apoptosis of islets beta cells. FBW7 destabilized EZH2 and accelerated ubiquitindependent degradation. EZH2 and H3K27me3 downregulated the ZBTB16 expression by accumulating in the ZBTB16 promoter and methylation.

\section{Conclusion}

FBW7 upregulates the expression of ZBTB16 by targeting histone methyltransferase EZH2 thus reducing the occurrence of T1D.

\section{Background}

Type I diabetes (T1D) is a heritable autoimmune disease [1]. Caused by autoimmune response against pancreatic beta cells, T1D results beta cell destruction and may accompanies with other autoimmune disease [2]. T1D is generally considered as a juvenile onset disease but it can occur at any age of life. Patients have to administrate insulin for survival causing tremendous burden to individuals and the society psychologically and economically [3]. T1D is a chronic autoimmune disease, which seriously impairs the pancreatic beta cells [4]. Healthcare provider are struggling for economic and standardized therapies. 
F-box and WD repeat domain-containing 7 (FBW7, also known as FBXW7), an E3 ubiquitin ligase, is found to be a pancreatic beta cell apoptosis inhibitor in vivo [5]. Of note, a recent study has suggested that FBW7 prevents the development of insulin resistance in the T2D mice models [6]. Despite that a previous genome wide association studies (GWAS) showed FBW7 contributed to the risk for type 2 diabetes (T2D) [7], evidence for the association of FBW7 and T1D is limited. These results suggest that FBW7 may play a role in diabetes and encourage us to investigate the functional relevance of FBW7 in T1D.

As a potential substrate of FBW7, enhancer of zeste homolog $2(\mathrm{EZH} 2)$ transfers a methyl group from $S$ adenosyl-L-methionine (SAM) to the lysine 27 on histone H3 [8]. Methylation by EZH2 assists the heterochromatin formation and suppresses the respective gene [9]. Interestingly, prior evidence has suggested that $\mathrm{EZH} 2$ was able to modulate pancreatic beta cell proliferation and regeneration in diabetes mellitus [10]. Recent report has documented that EZH2 can be suppressed by FBW7 in pancreatic cancer cells, thus restraining tumor cell migration and invasion [11]. Taken together, we reasonably propose EZH2 interacts with FBW7 and pancreatic cells in diabetes, which would fill the gap of FBW7 and T1D

Zinc finger and BTB domain containing 16 (ZBTB16, also known as PLZF), initially discovered as a cause of human retinoic acid-resistant acute promyelocytic leukemia, is a DNA sequence-specific transcriptional repressor [12]. Increasing studies have highlighted that ZBTB16 expression correlates with diabetes through the adaptive thermogenesis response, mitochondrial respiration promotion [13] and regulation of the insulin signaling pathway [14]. Importantly, ZBTB16 is found to be a possible candidate therapeutic target for T1D [15].

Here, we report that a regulatory axis of the three factors, FBW7, EZH2, and ZBTB16 contributes to the reduction of T1D occurrence. In this pathway, FBW7 degrades EZH2 and upregulates ZBTB16. We show that FBW7 is an emergent new therapeutic target for T1D treatment.

\section{Methods}

\section{Ethics statement}

The animal experimental processes were approved by the Ethnic Committee of Linyi People's Hospital and conducted in strict accordance to the Guide for the Care and Use of Laboratory Animals published by the US National Institutes of Health.

\section{Establishment of mouse model}

A total of 70 male NOD mice aged 4 weeks were obtained from Shanghai Eason Biotech Co., Ltd. (Shanghai, China). The mice were randomized into the normal group $(n=10)$ and T1D model group $(n=$ 60). For the streptozotocin (STZ)-induced T1D group, STZ (Sigma-Aldrich, Shanghai, China) at $40 \mathrm{mg} / \mathrm{kg}$ dose was administered intraperitoneally for 6 successive days. Nonfasting morning blood glucose concentrations were measured by tail vein prick using a OneTouch Ultra 2 blood glucose meter (LifeScan 
Inc., Milpitas, CA, USA) every other day. When two successive blood glucose readings were more than $250 \mathrm{mg} / \mathrm{dL}$, the mouse was considered as diabetic [16]. Afterwards, the occurrence was recorded every 6 days. The presented data were cumulative incidence of T1D collected from 3 independent experiments.

\section{Immunohistochemistry (IHC)}

The antigens were retrieved in a citrate repair solution. The tissue sections were subjected to blocking in a TBS solution containing $10 \%$ normal serum and $1 \%$ BSA for 2 hrs at room temperature. Each section was treated with antibodies at $4{ }^{\circ} \mathrm{C}$ overnight. Normal serum was employed as a negative control instead of primary antibody. Then, $50 \mu \mathrm{l}$ of $3 \% \mathrm{H}_{2} \mathrm{O}_{2}$ was added to each section, followed by incubation at room temperature for 20 min to eliminate endogenous peroxidase activity. Secondary antibody was added dropwise to each section, and incubated at $37^{\circ} \mathrm{C}$ for $30 \mathrm{~min}$. The sections were exposed to DAB reagent, and hematoxylin counterstaining. The brown color represented positive expression.

\section{Vector construction and cell transfection}

FBW7 and EZH2 coding region of the gene were ligated into PLVX-DsRed2-3FLAG vector. GFP Lentivirus control plasmid (Heyuan, Shanghai, China) was applied in this study. FBW7 shRNA, ZBTB16 shRNA, and negative control shRNA were obtained from Shanghai Gene Pharma Co., Ltd. (Shanghai, China) 293T cell was cultured and inoculated into petri dish $1 \mathrm{hr}$ prior to transfection. Lentiviral vectors containing pLVXFBW7 and pLVX-EZH2 were transfected to 293T cells.

After $24 \mathrm{hrs}$, the cell medium was changed to Dulbecco's Modified Eagle Medium (DMEM) supplemented with $10 \%$ feta bovine serum (FBS). After $48 \mathrm{hrs}$, the cells were centrifuged and the supernatant was collected, flited using a $0.45 \mu \mathrm{m}$ PVDF filter, and kept at $-80^{\circ} \mathrm{C}$. The plasmids were classified as shControl group, shFBW7-1 group, OE-NC group, OE-FBW7, OE-FBW7 + OE-NC group, OE-FBW7 + sh-NC group, OEFBW7 + OE-EZH2 group, and OE-FBW7 + sh-ZBTB16 group.

Lentiviral vectors with FBW7 overexpression, ZBTB16 interference, and control vectors were transduced into T1D mice via tail vein injection. The occurrence of T1D was determined after $24 \mathrm{hrs}$. The mice were sacrificed by cervical dislocation after 30 days. The pancreatic tissue, splenocyte, and pancreatic islet beta cells of mice were isolated [17]. Islet autoantigen glutamic acid decarboxylase (GAD65, $20 \mu \mathrm{g} / \mathrm{mL}$ ) was added to the splenocytes in vitro in order to induce T1D-specific T-cell response.

\section{Isolation of mouse islets}

Islets were isolated from the pancreas as follows. After occlusion of the distal end proximal to the duodenum, $4 \mathrm{~mL}$ collagenase $P$ solution with the concentration of $1 \mathrm{mg} / \mathrm{mL}$ was slowly injected into the common bile duct. The distended pancreas was excised. The digestion was performed in a water bath at $37^{\circ} \mathrm{C}$ for $30 \mathrm{~min}$. The digest was subjected to a Ficoll gradient separation to facilitate the harvesting of islets, which was then picked by hand and counted under an inverted microscope.

\section{Enzyme-linked immunosorbent assay (ELISA)}


To determine the cytokine levels, splenocytes were cultured in DMEM supplemented with 10\% FBS $(0.2 \mathrm{~mL})$ at a final concentration of $1.5 \times 10^{6}$ cells/ well. The supernatant was collected after $48 \mathrm{hrs}$ and cytokines were assessed using quantitative ELISA kit (BD Bioscience, San Jose, CA, USA) [18].

\section{Real-time polymerase chain reaction (RT-PCR)}

Total RNA was isolated using TRIzol (Invitrogen, Carlsbad, CA, USA) and reverse transcribed to cDNA using PrimeScript ${ }^{\text {TM }}$ RT Reagent Kit with gDNA Eraser (RR047A, Takara, Kusatsu, Japan). After a reverse transcription step at $37^{\circ} \mathrm{C}$ for $15 \mathrm{~min}$ followed by denaturation at $85^{\circ} \mathrm{C}$ for $5 \mathrm{sec}$, cDNA was mixed with TB Green Premix Ex Taq kit (RR420A, Takara, Kusatsu, Japan). Real-time PCR was conducted in ABI7500 system (Applied Biosystems, Foster City, CA, USA). Each sample was analyzed in triplicate. FBW7, EZH2, and ZBTB16 primer sequences were synthesized by Shanghai Sangon Biotech Co., Ltd (see Table 1 for primer sequence). CT values were recorded and GAPDH was served as the internal reference for FBW7, EZH2, and ZBTB16. The $2^{-\triangle \triangle C T}$ method was applied to calculate the relative expression of genes.

Table 1

Primer sequences for RT-qPCR

\begin{tabular}{|c|c|}
\hline Gene of interest & Sequence \\
\hline \multirow[t]{2}{*}{ FBW7 (human) } & Forward: 5'-CCACTGGGCTTGTACCATGTT-3' \\
\hline & Reverse: 5'-CAGATGTAATTCGGCGTCGTT-3' \\
\hline \multirow[t]{2}{*}{ FBW7 (mice) } & Forward: 5'-CGAGACTTCATCTCCTTGCTTCC-3' \\
\hline & Reverse: 5'-CCAGAGAAGGTTATCCTCAGCC-3' \\
\hline \multirow[t]{2}{*}{ EZH2 (human) } & Forward: 5'-GCCAGACTGGGAAGAAATCTG-3' \\
\hline & Reverse: 5'-TGTGCTGGAAAATCCAAGTCA-3' \\
\hline \multirow[t]{2}{*}{ EZH2 (mice) } & Forward: 5'-CAACCCGAAAGGGCAACAAA-3' \\
\hline & Reverse: 5'-TCACCAGTCTGGATAGCCCT-3' \\
\hline \multirow[t]{2}{*}{ ZBTB16 (human) } & Forward: 5'-GAGCTTCCTGATAACGAGGCTG-3' \\
\hline & Reverse: 5'-AGCCGCAAACTATCCAGGAACC-3' \\
\hline \multirow[t]{2}{*}{ ZBTB16 (mice) } & Forward: 5'-ACATACGGGTGTGAACTCTGCG-3' \\
\hline & Reverse: 5'-TGAGAACTGGGCACCGCATTGA-3' \\
\hline \multirow[t]{2}{*}{ GAPDH (human) } & Forward: 5'-САСССАСТССТССАССТTТG-3' \\
\hline & Reverse: 5'-CCACCACCCTGTTGCTGTAG-3' \\
\hline \multirow[t]{2}{*}{ GAPDH (mice) } & Forward: 5'-CATCACTGCCACCCAGAAGACTG-3' \\
\hline & Reverse: 5'-ATGCCAGTGAGCTTCCCGTTCAG-3' \\
\hline
\end{tabular}

\section{Western blot assay}


Tissue or cell total protein was extracted using RIPA lysis buffer (R0010, Solarbio, Beijing, China) at $4^{\circ} \mathrm{C}$ for $15 \mathrm{~min}$ followed by centrifugation at $15000 \mathrm{r} / \mathrm{min}$ for $15 \mathrm{~min}$ according to the user manual. The supernatant was collected, and the protein concentration was measured and normalized using bicinchoninic acid (BCA) kit (20201ES76, Yeasen, Shanghai, China). The proteins were separated by sodium dodecyl sulfate-polyacrylamide gel electrophoresis (SDS-PAGE), transferred onto polyvinylidene fluoride (PVDF) membrane, blocked in 5\% BSA for $1 \mathrm{hr}$. The membrane was washed with TBST buffer for 5 min three times, and incubated at $4{ }^{\circ} \mathrm{C}$ overnight with diluted primary antibodies: Fbxw7 (ab109617, 1:200), EZH2 (ab186006, 1:500), ZBTB16 (ab39354, 1:1000), FLAG (ab1162, 1:200), HA (ab9110, 1:2000), Myc (ab9106, 1:500), Ub (ab7780, 1:500). All the rabbit polyclonal antibodies were purchased from Abcam, Cambridge, UK unless otherwise noted.

After incubation, the membrane was washed with TBST buffer for 5 min three times. HRP labeled antiRabbit IgG H\&L (ab205718, 1:10000) was added at room temperature. The membrane was incubated for $1 \mathrm{hr}$ at room temperature followed by TBST wash for $10 \mathrm{~min}$ three times. The blot was visualized with enhanced chemiluminescence (ECL) regents. The images were captured using FUSION FX5 (Vilber Lourmar, France), and analyzed by ImageJ 1.48u (National Institutes of Health, USA). GAPDH (ab9485, 1:500) was served as the internal reference. Each experiment was conducted in triplicate [18].

\section{Chromatin immunoprecipitation (ChIP) assay}

ChIP assay was performed to determine the accumulation of EZH2 and H3K27me3 in the ZBTB16 promoter region using the EZ-Magna ChIP kit (17-10086, Millipore, Billerica, MA, USA). Airway smooth muscle cells (ASMCs) in the logarithmic growth phase were crosslinked with formaldehyde at the final concentration of $1 \%$ for $10 \mathrm{~min}$. Crosslinking was quenched by the addition of $125 \mathrm{mM}$ glycine at room temperature for $5 \mathrm{~min}$. Cells were washed with pre-cooled PBS buffer twice, collected after centrifuging for $5 \mathrm{~min}$ at $2000 \mathrm{rpm}$, and suspended in the lysis buffer with the concentration of $150 \mathrm{mM} \mathrm{NaCl}, 50 \mathrm{mM}$ Tris ( $\mathrm{pH}=7.5), 5 \mathrm{mM}$ EDTA, $0.005 \%$ NP40, $0.01 \%$ Triton $X-100$. The final concentration of cells was $2 \times$ $10^{6}$ per $200 \mathrm{~mL}$. After adding the protease inhibitors, the mixture was centrifuged at $5000 \mathrm{rpm}$ for $5 \mathrm{~min}$. The cell pellet was resuspended in nuclear isolation buffer, lysed on ice for $10 \mathrm{~min}$, sheared to 200$1,000 \mathrm{bp}$ by sonication, and centrifuged at $14000 \times \mathrm{g}$ at $4^{\circ} \mathrm{C}$ for $10 \mathrm{~min}$. The supernatant $(100 \mu \mathrm{L}), \mathrm{ChIP}$ dilution buffer $(900 \mu \mathrm{L})$, protease inhibitor cocktail (PIC, 50x, $20 \mu \mathrm{L})$, and Protein A-Agarose/Salmon Sperm DNA (60 $\mu \mathrm{L}$, Sigma-Aldrich, Shanghai, China) were mixed at $4^{\circ} \mathrm{C}$ for $1 \mathrm{hr}$ and allowed to stand still at same temperature for $10 \mathrm{~min}$. The mixture was centrifuged at $700 \mathrm{rpm}$ for $1 \mathrm{~min}$. The supernatant (20 $\mu \mathrm{L})$ from experimental group was mixed with EZH2 Ab (1 $\mu \mathrm{L}$, ab186006, 1:200), H3K27me3 Ab $(1 \mu \mathrm{L}$, ab192985, 1:200), and Protein A-Agarose/Salmon Sperm DNA (60 $\mu \mathrm{L})$. For the control experiments, rabbit IgG $(1 \mu \mathrm{L}, \mathrm{zb} 186006,1: 200)$ was used. The system was incubated at $4^{\circ} \mathrm{C}$ for $2 \mathrm{hrs}$ and allowed to stand still at the same temperature for $10 \mathrm{~min}$ followed by centrifugation at $700 \mathrm{rpm}$ for $1 \mathrm{~min}$. The supernatant was discarded. The precipitate was washed with low salt buffer $(1 \mathrm{~mL})$, high salt buffer $(1 \mathrm{~mL})$, LiCl aq. $(1 \mathrm{~mL})$, TE buffer $(1 \mathrm{~mL}, 2 \times)$. ChIP wash buffer $(250 \mu \mathrm{L}, 2 \times)$ was added to elute the complex. DNA was decrosslinked and recycled by adding $\mathrm{NaCl}$ aq. $(5 \mathrm{M}, 20 \mu \mathrm{L})$ in which RT-PCR was performed to quantify the LRP5 promoter [19]. 


\section{Protein stability assay}

Vector shControl and shFBW7 were transfected into Min6 cells per calcium phosphate method. After 24 hrs, the cells were treated with cycloheximide (CHX, CalBiochem, Gibbstown, NJ, USA) at a dose of $40 \mu \mathrm{g} /$ $\mathrm{mL}$. The cells were harvest and lyzed at designed time points. The lysate was analyzed by Western blot, imaged and quantified by Licor Odyssey and Image Quant software [20,21].

\section{Flow cytometry}

Apoptosis of pancreatic beta cells was detected by flow cytometry. Pancreatic beta cells were isolated from T1D mice and cultured in the medium supplemented with TNF-a $(10 \mathrm{ng} / \mathrm{mL})$ for 3 days to simulate the insulin-dependent T1D [17]. The medium was discard and the cells were washed with PBS buffer twice and centrifuged. The cell pellet was resuspended in the binding buffer to give a single cell suspension with a concentration of $5 \times 10^{5}$ cell/mL. Annexin $\mathrm{V}(5 \mu \mathrm{L})$ and 7-aminoactinomycin $\mathrm{D}(7 \mathrm{AAD}$, $5 \mu \mathrm{L}$ ) were added to the suspension at room temperature protected from light. The mixture was incubated for 30 min before determined by flow cytometry (BD Bioscience, San Jose, CA, USA).

\section{Assessment of cytotoxicity by CCK-8 assays}

Cells were treated with the same protocol as stated in the flow cytometry. Cytotoxicity was checked using the CCK-8 kit (Yisen, Shanghai, China). First, the cell suspension was placed in a 96-well plate with a concentration of $5 \times 10^{4}$ cell/well, and pre-warmed in the incubator $\left(37^{\circ} \mathrm{C}, 5 \% \mathrm{CO}_{2}\right)$. Next, CCK-8 $(10 \mu \mathrm{L})$ was added to each well and the plate was incubated for 1-4 hrs. Finally, the absorbance at $450 \mathrm{~nm}$ was recorded on a microplate reader (Bio-Rad, Hercules, CA, USA) [18].

\section{Assessment of insulin secretion}

Min6 and pancreatic beta cells were inoculated on a 24-well plate at the concentration of $5 \times 10^{4}$ cell/well pretreated with or without Notoginsenoside R1 (NGR1). Next, the pancreatic beta cells were placed in the medium supplemented with TNF-a $(20 \mathrm{ng} / \mathrm{mL})$ for 3 days to simulate the islet inflammation in T1D. The supernatant was collected and the insulin concentration was determined by radioimmunoassay [17, 22].

\section{Statistical analysis}

Statistical and mathematical data were processed by SPSS 21.0. Values were reported as mean \pm standard deviation. Data comparisons between two groups were analyzed by unpaired $t$-test. Comparisons among multiple groups were performed by one-way analysis of variance (ANOVA). Statistical analysis in relation to time-based measurements within each group was realized using repeated measures ANOVA. A value of $p<0.05$ indicated significant difference.

\section{Results}

\section{FBW7 is under-expressed in mouse model with T1D}


The correlation between FBW7 and diabetic nephropathy has been previously established [23]. T1D mouse model was established using STZ. After 15 days of STZ administration, averaged blood glucose levels of the mice models exceeded $250 \mathrm{mg} / \mathrm{dL}$, which confirmed the T1D model has been successfully established (Fig. 1A). FBW7 expression levels in the pancreatic islets of mice were examined by Western blot assay and IHC. A significant decrease in the FBW7 expression level was observed in the T1D model group (Fig. 1B and C). The above results concluded that FBW7 was down-regulated in T1D mouse model.

\section{FBW7 suppresses the occurrence of T1D in vivo and in vitro}

To further illustrate the impact of FBW7 on T1D, we overexpressed FBW7 in the T1D mousee model and analyzed the incidence of T1D in both groups. Compared to the normal group, T1D incidence was increased in the model group. Compared to the overexpression negative control (OE-NC) group, T1D incidence declined noticeably in the overexpression FBW7 (OE-FBW7) group (Fig. 2A). The siz of the islet was measured after isolation. Results showed that mice islets were remarkably shrunken in the model group in comparison with the normal group while islets were expanded in the OE-FBW7 group as to the OE-NC group (Fig. 2B). FBW7 expression was identified using Western blot (Fig. 2C). Compare to the normal group, FBW7 expression in the mice islet from the model group was negatively regulated. The expression level in the mice islets of the OE-FBW7 group was positively regulated as to the OE-NC group. Therefore, we showed FBW7 decreased the T1D incidence in vivo.

Autoreactive T cells are considered as the major factor in the beta cell destruction. Therefore, we simulated the T1D-induced isletitis by mixing splenocytes and T1D-specific T cells and induced Min6 apoptosis in beta cells using inflammatory factor TNF-a. FBW7 was over-expressed in a part of cells from the model group. FBW7 expression in the splenocytes and pancreatic beta cells were checked by Western blot. We observed that FBW7 in both cell types from the model group was down-regulated compared to the normal group. Compared to the OE-NC group, FBW7 was reasonably up-regulated in both cells type from the OE-FBW7 group (Fig. 2D). ELISA was applied to measure the IL-17IL-2IIFN-yIL-4 level in the cell supernatant (Fig. 2E). All four cytokines production has been elevated in the model group compared to the normal group while all have been depressed in the OE-FBW7 group with reference to the OE-NC group. These results demonstrated FBW7 can eliminate the activation of autoreactive T cells thus preventing T1D.

Additionally, pancreatic beta cell apoptosis was verified by flow cytometry (Fig. 2F). Apoptosis was accelerated in the model group relative to the normal group. Refer to the OE-NC group, apoptosis in the OE-FBW7 group decreased considerably. Assessment of glucose-stimulated insulin secretion disclosed that insulin secretion was lessened in the model group compared to the normal group while enhanced secretion was observed in the OE-FBW7 group in refer to the OE-NC group (Fig. 2G). Here, we have shown FBW7 decreased the T1D incidence both in vitro and in vivo.

\section{FBW7 inhibits TNF-a-induced pancreatic beta cell apoptosis and dysfunction through ubiquitin degradation of EZH2}


T1D downstream pathway affected by FBW7 was further investigated. Researchers reported that EZH2, highly expressed in T1D rat [24], was a substrate of FBW7 and was negatively regulated [11].

To clarify whether FBW7 acts as E3 ubiquitin ligase of EZH2, we transfected the FBW7 vector with Myc tag in gradually increased concentration, or empty vector, into Min6 cells, together with Flag-tagged EZH2 vector at a constant concentration. After $24 \mathrm{hrs}$, the cells were incubated with MG132, a proteasome inhibitor, or dimethyl sulfoxide (DMSO) for $16 \mathrm{hrs}$. Western blot revealed that ectopically expressed EZH2 was co-downregulated by FBW7 while under-expression of EZH2 was blocked by MG132 (Fig. 3A). We have tested the effect of FBW7 in endogenous EZH2 in pancreatic beta cells. Knocking down the endogenous FBW7 by two independent Fbw7-specific shRNAs enhanced the EZH2 expression level (Fig. 3B), leaving EZH2 mRNA expression unchanged (Fig. 3C). Moreover, Knocking down Fbw7 increased the stability of endogenous EZH2 as shown in Western blot (Fig. 3D). Results obtained from immunoprecipitation demonstrated EZH2 polyubiquitination was diminished as $\mathrm{Fbw} 7$ was knocked down (Fig. 3E). The results above indicate FBW7 boosted EZH2 ubiquitination and proteasome degradation.

Next, we explained the effect of the FBW7 and EZH2 on the TNF-a-induced pancreatic beta cells. FBW7 alone, or together with EZH2, was overexpressed in Min6 cells. As shown in Western blot, FBW7 was upregulated impressively while EZH2 was downregulated, which was then remedied by EZH2 overexpression (Fig. 3F). Cytotoxicity assay with CCK-8 and flow cytometry revealed that the OE-FBW7+ OE-NC group displayed higher cell viability and less apoptosis as to the OE-NC group while overexpression EZH2 simultaneously caused more cytotoxicity and accelerated apoptosis (Fig. 3G and $3 \mathrm{H})$. Assessment of glucose-induced insulin secretion proved that more insulin was secreted from the OEFBW7 + OE-NC group as to the OE-NC group while secretion from OE-FBW7 + OE-EZH2 group was uprooted (Fig. 3I). In short, we settled down with the conclusion that TNF-a-induced beta cells apoptosis and dysfunction was inhibited by FBW7 through ubiquitin degradation of EZH2.

\section{EZH2 enhances TNF-a-induced pancreatic beta cells apoptosis and dysfunction through ZBTB16 methylation}

Bioinformatic analysis revealed ZBTB16 was down-regulated in T1D patients and EZH2 suppressed ZBTB16 expression [19]. Min6 cell model was established by EZH2 overexpression, or with ZBTB16 overexpression simultaneously, and TNF- $\mathrm{a}$ induction. Western blot convinced the positive regulation of EZH2 and negative regulation of ZBTB16 in EZH2-overexpressed Min6 cells. Overexpression of ZBTB16 reversed its expression (Fig. 4A).

Considering the possibility of Zbtb16 methylation, ChIP assay was performed to testify the enrichment of EZH2 and H3K27me3 on Zbtb16 (Fig. 4B and C). EZH2 and EZH2-mediated H3K27me3 were accumulated in the $Z$ btb16 promoter region.

Furthermore, cell viability has been tested using the CCK-8 kit. OE-EZH2 + OE-NC group showed worse cell viability compared to both the OE-NC group and the OE-EZH2 + OE-ZBTB16 group (Fig. 4D). Apoptosis was monitored by flow cytometry (Fig. 4E). The bivariate histogram displayed the apoptosis was 
accelerated in the OE-EZH2 + OE-NC group compared to the OE-NC group. Apoptosis had been retarded in the OE-EZH2 + OE-ZBTB16 group as to the OE-EZH2 + OE-NC group. Glucose-induced insulin secretion assay proved that insulin secreted more in the OE-EZH2 + OE-NC group as to the OE-NC group while insulin secretion from OE-EZH2 + OE-ZBTB16 group was eliminated refer to the OE-EZH2 + OE-NC group (Fig. 4F). Therefore, we were confident to conclude that EZH2 was anti-correlated to ZBTB16. EZH2 mediated Zbtb16 methylation thus enhancing TNF-a-induced pancreatic beta cells apoptosis and dysfunction.

\section{FBW7 inhibits T1D occurrence via EZH2/ZBTB16 in vivo and in vitro}

To verify the EZH2/ZBTB16 pathway in the T1D inhibition by FBW7, we transfected the shZBTB16 vector to the Min6 cells. The following experiments were conducted using sh-ZBTB16-1 cells selected by qRTPCR (Fig. 5A).

T1D mouse model with Fbw7 overexpression, or simultaneously with Zbtb16 suppression using shRNA, were established and checked for diabetes incidence (Fig. 5B). Compared to the OE-FBW7 + sh-NC group, the OE-FBW7 + sh-ZBTB16 group showed a significantly higher occurrence of T1D. Islets were isolated and their sizes were measured and plotted (Fig. 5C). Islets from the OE-FBW7 + sh-ZBTB16 group shrank considerably referred to the OE-FBW7 + sh-NC group. Western bolt has been done to determine the expression of FBW7, EZH2, and ZBTB16 in the islet (Fig. 5D). Expression levels of FBW7 and ZBTB16 were escalated while EZH2 declined in the OE-FBW7 + sh-NC group as to the OE-NC group. In comparison to the OE-FBW7 + sh-NC group, no significant change in FBW7 and EZH2 expression but a reduction in ZBTB16 expression was observed. From the above, we have shown the EZH2/ZBTB16 pathway played a role in T1D inhibition of FBW7 in vivo.

Meanwhile, mice splenocytes were reacted with T1D-specific T cells. A part of model cells overexpressed FBW7, or simultaneously suppressed Zbtb16. Pancreatic beta cell Min6 was induced to apoptosis by inflammatory factor TNF-a to simulate the isletitis T1D. Expression of FBW7, EZH2, and ZBTB16 in the splenocytes and pancreatic beta cells were revealed by Western blot (Fig. 5E). Compared to the OE-NC+ sh-NC group, FBW7 and ZBTB16 were positively regulated while EZH2 was negatively regulated in the OEFBW7 + sh-NC group. Refer to the OE-FBW7 + sh-NC group, expression of FBW7 and EZH2 stayed consistent while ZBTB16 decreased in the OE-FBW7 + sh-ZBTB16 group. Quantification of IL-17LIL-2IIFNYIIL-4 in the cell supernatant was achieved by ELISA (Fig. 5F). All four cytokines were boosted in Zbtb16silenced cells compared to the OE-FBW7 + sh-NC group. Determination of pancreatic beta cell apoptosis by flow cytometry indicated expedited apoptosis occurred in the OE-FBW7 + sh-ZBTB16 group refer to OEFBW7 + sh-NC group (Fig. 5G). Assessment of glucose-induced insulin secretion confirmed that less insulin was secreted in the OE-FBW7 + sh-ZBTB16 group as to OE-FBW7 + sh-NC (Fig. 5H). In conclusion, sufficient evidence is found to claim that FBW7 inhibited T1D occurrence via the EZH2/ZBTB16 pathway both in vivo and in vitro.

\section{Discussion}


In the past decades, our knowledge on T1D increased rapidly, but insufficient to satisfy the demands on standardized clinical treatment and contracting disease-associated burdens. Researches on T1D were concentrated on the mechanism of autoimmunity [25] and genetically relevant loci in human leukocyte antigen (HLA) [26, 27], which might benefit for T1D prediction and prevention [28]. Here in the present study, the mechanism of EZH2/ZBTB16 in T1D inhibition by FBW7 is clarified, suggesting that FBW7 is a promising target for T1D therapy.

Previous GWAS showed that more than 40 loci link to the risk of T1D [29], a few of which are non-HLA loci, e.g. INS, CTLA4, and PTPN22. We are interested in these non-HLA loci as HLA loci possibly associated with other autoimmune diseases. We started our further investigation from FBW7 located on chromosome 4, encoding an E3 ubiquitin ligase that targets key regulators of cell division and growth for ubiquitylation and subsequent degradation by the proteasome [30]. FBW7 suppresses colorectal cancer [31,32], gastric cancer [33], and pancreatic cancer [34] through ubiquitin degradation of varied components in the pathway. The experimental data obtained from our study validated that FBW7 was downregulated both in T1D mouse model. Growing evidence has implied that F-box protein protects pancreatic beta cells and negatively regulates the cytokines in renal mesangial cells $[23,35]$. Results in the present study confirmed that FBW7 impeded the T1D incurrence, corresponding to down-regulated cytokines expression and inhibited pancreatic beta cell apoptosis, indicating its significance in autoimmune diseases.

A wide variety of oncogenic proteins are found to be the substrates of FBW7 for ubiquitination including c-Myc [36], SREBP [37], and HIF-1a [32], many of which play key roles in metabolic pathways [38]. We found EZH2 was the FBW7 substrate as we investigated the downstream pathway. Similarly, FBW7dependent ubiquitination and degradation of EZH2 is found in the pancreatic cancer cells and prevents cancer migration and invasion [11]. EZH2 degradation by FBW7 is meanwhile reported in macrophage [39]. These results strongly suggest the important function of FBW7 in the regulation of the EZH2 protein level.

As the enzymatic subunit of Polycomb Repressive Complex 2 (PRC2), EZH2 epigenetically silences the genes in target promoters by histone $\mathrm{H} 3$ lysine 27 trimethylation [40]. In addition to H3K27me3, EZH2 can also bind DNMT1 thus methylating DNA [41]. Kowluru et al. reported that DNMT1 alters the methylation status of MMP-9 promoter in diabetes $[42,43]$. Studies on the parallel possible methylation pathway, as well as determination of $5 \mathrm{mC}$ and $5 \mathrm{hmC}$ levels at the ZBTB16 promoter, are currently ongoing in our lab.

ZBTB16 has been proved to beneficial for tumor suppression as it represses c-myc oncogene [44]. ZBTB16 is critical in diabetes progression. Activation of ZBTB16 reduces serum advanced glycation end (AGE) products in STZ-induced diabetic mice and further inhibits AGE-induced apoptosis [45]. Furthermore, ZBTB16 involves in insulin signaling pathway regulation by reduction of phosphorylation levels of IRS1, Ake, and FoxO1 in the mouse model [46]. In our study, we described ZBTB16 negatively correlated to pancreatic beta cell apoptosis and contributed to beta cell viability. Therefore, the downstream pathway of T1D inhibition by FBW7 has been established. 


\section{Conclusions}

Taken together, this study suggests the role of FBW7 (Fig. 6), which sits outside the HLA region, in the prevention of T1D occurrence. We established the FWB7/EZH2/ZBTB16 axis in the T1D pathogenesis and provided a new therapeutic target for T1D treatment. Further research should focus on other T1Drelated genes and the detailed interaction of ZBTB16 and pancreatic beta cell apoptosis and dysfunction.

\section{Abbreviations}

T1D: Type I diabetes; NOD: non-obese diabetic; IP: immunoprecipitation; ChIP: chromatin immunoprecipitation; GWAS: genome wide association studies; EZH2: enhancer of zeste homolog 2; SAM: S-adenosyl-L-methionine; BCA: bicinchoninic acid; PVDF: polyvinylidene fluoride.

\section{Declarations}

\section{Authors' contributions}

JMT and YYZ designed the study. JMT, YYZ, SSL performed the experiment. YYZ and SSL analysed the data and wrote the manuscript. JMT, SSL participated in the design of the study and helped to draft the manuscript. YYZ and SSL helped to draft the manuscript and participated in the data analysis. All authors read and approved the final manuscript.

\section{Author details}

1 Department of Pediatrics, Linyi People's Hospital, Linyi 276000, P.R. China

2 Department of Endocrinology, Linyi People's Hospital, Linyi 276000, P.R. China

\section{Acknowledgments}

The authors would like to acknowledge the helpful comments on this paper received from the reviewers.

\section{Competing interests}

The authors declare that they have no competing interests

\section{Availability of data and materials}

Data sharing not applicable to this article as no datasets were generated or analysed during the current study.

\section{Ethics approval and consent to participate}

The animal experimental processes were approved by the Ethnic Committee of Linyi People's Hospital and conducted in strict accordance to the Guide for the Care and Use of Laboratory Animals published by 
the US National Institutes of Health.

\section{Funding}

None.

\section{Consent for publication}

Not applicable

\section{References}

1. Thomas NJ, Jones SE, Weedon MN, Shields BM, Oram RA, Hattersley AT. Frequency and phenotype of type 1 diabetes in the first six decades of life: a cross-sectional, genetically stratified survival analysis from UK Biobank. Lancet Diabetes Endocrinol. 2018;6:122-9.

2. Kahaly GJ, Hansen MP. Type 1 diabetes associated autoimmunity. Autoimmun Rev. 2016;15:644-8.

3. Prahalad P, Tanenbaum M, Hood K, Maahs DM. Diabetes technology: improving care, improving patient-reported outcomes and preventing complications in young people with Type 1 diabetes. Diabet Med. 2018;35:419-29.

4. Pugliese A. Autoreactive T cells in type 1 diabetes. J Clin Invest. 2017;127:2881-91.

5. Meyerovich K, Fukaya M, Terra LF, Ortis F, Eizirik DL, Cardozo AK. The non-canonical NF-kappaB pathway is induced by cytokines in pancreatic beta cells and contributes to cell death and proinflammatory responses in vitro. Diabetologia. 2016;59:512-21.

6. Zhao J, Xiong X, Li Y, Liu X, Wang T, Zhang H, Jiao Y, Jiang J, Zhang H, Tang Q, et al. Hepatic F-Box Protein FBXW7 Maintains Glucose Homeostasis Through Degradation of Fetuin-A. Diabetes. 2018;67:818-30.

7. Imamura M, Takahashi A, Yamauchi T, Hara K, Yasuda K, Grarup N, Zhao W, Wang X, Huerta-Chagoya $\mathrm{A}$, Hu C, et al. Genome-wide association studies in the Japanese population identify seven novel loci for type 2 diabetes. Nat Commun. 2016;7:10531.

8. Cao R, Wang L, Wang H, Xia L, Erdjument-Bromage H, Tempst P, Jones RS, Zhang Y. Role of histone H3 lysine 27 methylation in Polycomb-group silencing. Science. 2002;298:1039-43.

9. Vire E, Brenner C, Deplus R, Blanchon L, Fraga M, Didelot C, Morey L, Van Eynde A, Bernard D, Vanderwinden JM, et al. The Polycomb group protein EZH2 directly controls DNA methylation. Nature. 2006;439:871-4.

10. Chen H, Gu X, Su IH, Bottino R, Contreras JL, Tarakhovsky A, Kim SK. Polycomb protein Ezh2 regulates pancreatic beta-cell Ink4a/Arf expression and regeneration in diabetes mellitus. Genes Dev. 2009;23:975-85.

11. Jin X, Yang C, Fan P, Xiao J, Zhang W, Zhan S, Liu T, Wang D, Wu H. CDK5/FBW7-dependent ubiquitination and degradation of EZH2 inhibits pancreatic cancer cell migration and invasion. $\mathrm{J}$ Biol Chem. 2017;292:6269-80. 
12. Grignani F, De Matteis S, Nervi C, Tomassoni L, Gelmetti V, Cioce M, Fanelli M, Ruthardt M, Ferrara FF, Zamir l, et al. Fusion proteins of the retinoic acid receptor-alpha recruit histone deacetylase in promyelocytic leukaemia. Nature. 1998;391:815-8.

13. Plaisier CL, Bennett BJ, He A, Guan B, Lusis AJ, Reue K, Vergnes L. Zbtb16 has a role in brown adipocyte bioenergetics. Nutr Diabetes. 2012;2:e46.

14. Chen S, Qian J, Shi X, Gao T, Liang T, Liu C. Control of hepatic gluconeogenesis by the promyelocytic leukemia zinc finger protein. Mol Endocrinol. 2014;28:1987-98.

15. Jia X, Yu H, Zhang H, Si Y, Tian D, Zhao X, Luan J, Jia H. Integrated analysis of different microarray studies to identify candidate genes in type 1 diabetes. J Diabetes. 2017;9:149-57.

16. Mathews CE, Xue S, Posgai A, Lightfoot YL, Li X, Lin A, Wasserfall C, Haller MJ, Schatz D, Atkinson MA. Acute Versus Progressive Onset of Diabetes in NOD Mice: Potential Implications for Therapeutic Interventions in Type 1 Diabetes. Diabetes. 2015;64:3885-90.

17. Ruan Q, Wang T, Kameswaran V, Wei Q, Johnson DS, Matschinsky F, Shi W, Chen YH. The microRNA21-PDCD 4 axis prevents type 1 diabetes by blocking pancreatic beta cell death. Proc Natl Acad Sci U S A. 2011;108:12030-5.

18. Hao Y, Wang W, Wu D, Liu K, Sun Y. Bilobalide alleviates tumor necrosis factor-alpha-induced pancreatic beta-cell MIN6 apoptosis and dysfunction through upregulation of miR-153. Phytother Res. 2020;34:409-17.

19. Hemming S, Cakouros D, Vandyke K, Davis MJ, Zannettino AC, Gronthos S. Identification of Novel EZH2 Targets Regulating Osteogenic Differentiation in Mesenchymal Stem Cells. Stem Cells Dev. 2016;25:909-21.

20. Wang YC, Wu YS, Hung CY, Wang SA, Young MJ, Hsu TI, Hung JJ. USP24 induces IL-6 in tumorassociated microenvironment by stabilizing p300 and beta-TrCP and promotes cancer malignancy. Nat Commun. 2018;9:3996.

21. Colleran A, Collins PE, O'Carroll C, Ahmed A, Mao X, McManus B, Kiely PA, Burstein E, Carmody RJ. Deubiquitination of NF-kappaB by Ubiquitin-Specific Protease-7 promotes transcription. Proc Natl Acad Sci U S A. 2013;110:618-23.

22. Suk K, Kim S, Kim YH, Kim KA, Chang I, Yagita H, Shong M, Lee MS. IFN-gamma/TNF-alpha synergism as the final effector in autoimmune diabetes: a key role for STAT1/IFN regulatory factor-1 pathway in pancreatic beta cell death. J Immunol. 2001;166:4481-9.

23. Gao C, Fan F, Chen J, Long Y, Tang S, Jiang C, Xu Y. FBW7 Regulates the Autophagy Signal in Mesangial Cells Induced by High Glucose. Biomed Res Int. 2019;2019:6061594.

24. Komers R, Mar D, Denisenko O, Xu B, Oyama TT, Bomsztyk K. Epigenetic changes in renal genes dysregulated in mouse and rat models of type 1 diabetes. Lab Invest. 2013;93:543-52.

25. Kroger CJ, Clark M, Ke Q, Tisch RM. Therapies to Suppress beta Cell Autoimmunity in Type 1 Diabetes. Front Immunol. 2018;9:1891.

26. Cain MT, Jones MC, Reed DN. A Novel Technique to Repair Acute and Chronic Proximal Hamstring Avulsions. Orthopedics. 2018;41:116-9. 
27. Styka AN, White DS, Zumwalt RE, Lathrop SL. Trends in adult suicides in New Mexico: utilizing data from the New Mexico violent death reporting system. J Forensic Sci. 2010;55:93-9.

28. Redondo MJ, Geyer S, Steck AK, Sharp S, Wentworth JM, Weedon MN, Antinozzi P, Sosenko J, Atkinson M, Pugliese A, et al. A Type 1 Diabetes Genetic Risk Score Predicts Progression of Islet Autoimmunity and Development of Type 1 Diabetes in Individuals at Risk. Diabetes Care. 2018;41:1887-94.

29. Davies MJ, Rode J, Woolf N, Krikler DM. Neuroendocrine differentiation in atrial myxomas. Lancet. 1987;2:800.

30. Welcker M, Clurman BE. FBW7 ubiquitin ligase: a tumour suppressor at the crossroads of cell division, growth and differentiation. Nat Rev Cancer. 2008;8:83-93.

31. Khan OM, Carvalho J, Spencer-Dene B, Mitter R, Frith D, Snijders AP, Wood SA, Behrens A. The deubiquitinase USP9X regulates FBW7 stability and suppresses colorectal cancer. J Clin Invest. 2018;128:1326-37.

32. Li Q, Li Y, Li J, Ma Y, Dai W, Mo S, Xu Y, Li X, Cai S. FBW7 suppresses metastasis of colorectal cancer by inhibiting HIF1alpha/CEACAM5 functional axis. Int J Biol Sci. 2018;14:726-35.

33. Huang LY, Zhao J, Chen H, Wan L, Inuzuka H, Guo J, Fu X, Zhai Y, Lu Z, Wang X, et al. SCF(FBW7)mediated degradation of Brg1 suppresses gastric cancer metastasis. Nat Commun. 2018;9:3569.

34. Ji S, Qin Y, Shi S, Liu X, Hu H, Zhou H, Gao J, Zhang B, Xu W, Liu J, et al. ERK kinase phosphorylates and destabilizes the tumor suppressor FBW7 in pancreatic cancer. Cell Res. 2015;25:561-73.

35. Gorrepati KDD, He W, Lupse B, Yuan T, Maedler K, Ardestani A. An SCF(FBXO28) E3 Ligase Protects Pancreatic beta-Cells from Apoptosis. Int J Mol Sci. 2018;19.

36. Zhang Z, Liu M, Hu Q, Xu W, Liu W, Sun Q, Ye Z, Fan G, Xu X, Yu X, et al. FGFBP1, a downstream target of the FBW7/c-Myc axis, promotes cell proliferation and migration in pancreatic cancer. Am J Cancer Res. 2019;9:2650-64.

37. Bengoechea-Alonso MT, Ericsson J. The phosphorylation-dependent regulation of nuclear SREBP1 during mitosis links lipid metabolism and cell growth. Cell Cycle. 2016;15:2753-65.

38. Shimizu K, Nihira NT, Inuzuka H, Wei W. Physiological functions of FBW7 in cancer and metabolism. Cell Signal. 2018;46:15-22.

39. He J, Song Y, Li G, Xiao P, Liu Y, Xue Y, Cao Q, Tu X, Pan T, Jiang Z, et al. Fbxw7 increases CCL2/7 in CX3CR1hi macrophages to promote intestinal inflammation. J Clin Invest. 2019;130:3877-93.

40. Zhao JC, Yu J, Runkle C, Wu L, Hu M, Wu D, Liu JS, Wang Q, Qin ZS, Yu J. Cooperation between Polycomb and androgen receptor during oncogenic transformation. Genome Res. 2012;22:322-31.

41. Wong CM, Wong CC, Ng YL, Au SL, Ko FC, Ng IO. Transcriptional repressive H3K9 and H3K27 methylations contribute to DNMT1-mediated DNA methylation recovery. PLoS One. 2011;6:e16702.

42. Kowluru RA, Shan Y, Mishra M. Dynamic DNA methylation of matrix metalloproteinase-9 in the development of diabetic retinopathy. Lab Invest. 2016;96:1040-9. 
43. Duraisamy AJ, Mishra M, Kowluru RA. Crosstalk Between Histone and DNA Methylation in Regulation of Retinal Matrix Metalloproteinase-9 in Diabetes. Invest Ophthalmol Vis Sci. 2017;58:6440-8.

44. Panitsas KE, Boyd CA, Meredith D. Evidence that the rabbit proton-peptide co-transporter PepT1 is a multimer when expressed in Xenopus laevis oocytes. Pflugers Arch. 2006;452:53-63.

45. Chen $\mathrm{CH}$, Chen TH, Wu MY, Chou TC, Chen JR, Wei MJ, Lee SL, Hong LY, Zheng CM, Chiu IJ, et al. Farinfrared protects vascular endothelial cells from advanced glycation end products-induced injury via PLZF-mediated autophagy in diabetic mice. Sci Rep. 2017;7:40442.

46. Koutenaei BA, Guler O, Wilson E, Thoranaghatte RU, Oetgen M, Navab N, Cleary K. Improved screw placement for slipped capital femoral epiphysis (SCFE) using robotically-assisted drill guidance. Med Image Comput Comput Assist Interv. 2014;17:488-95.

\section{Figures}

A
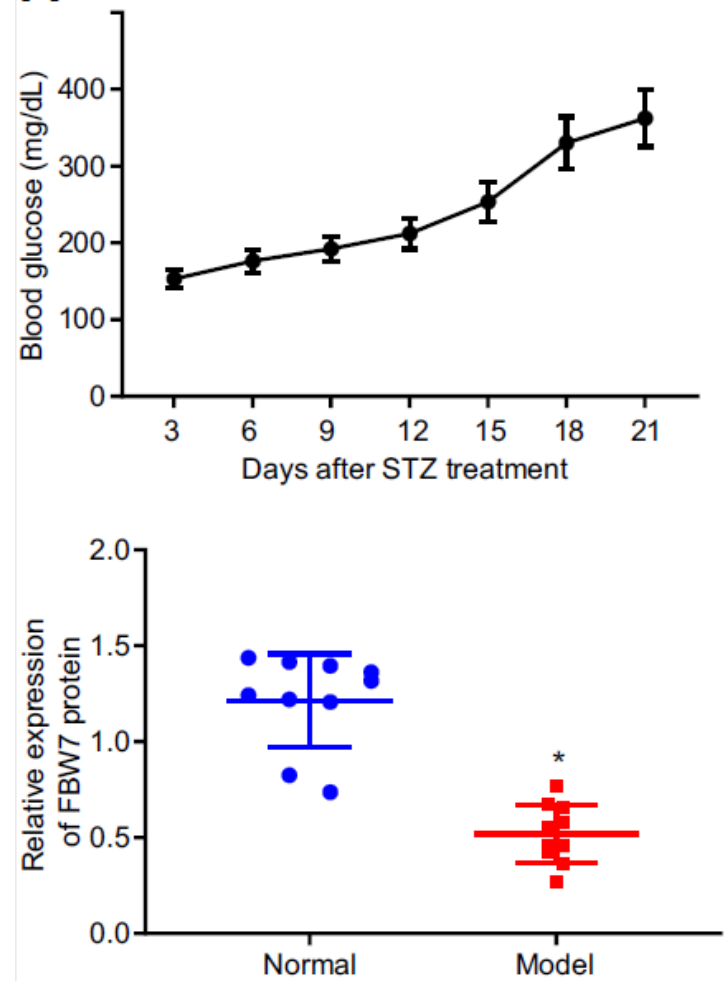

B

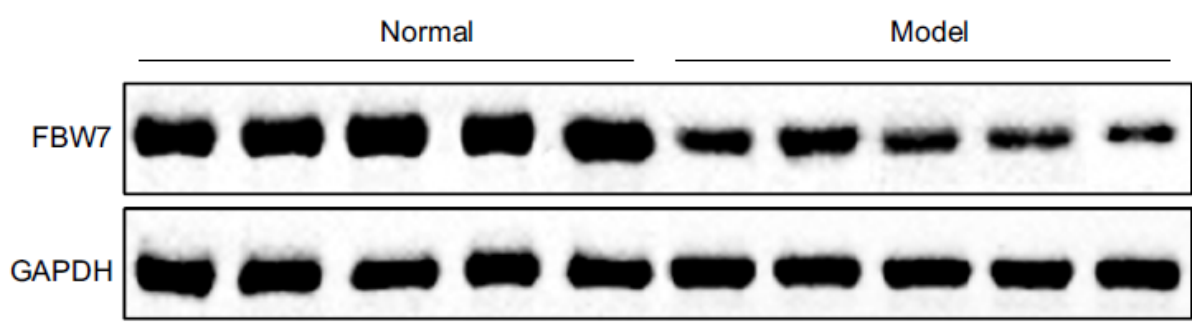

C

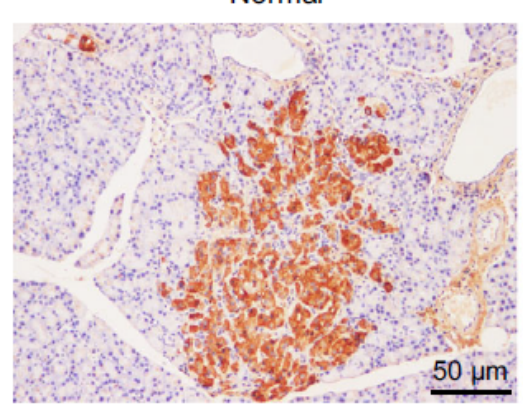

Model

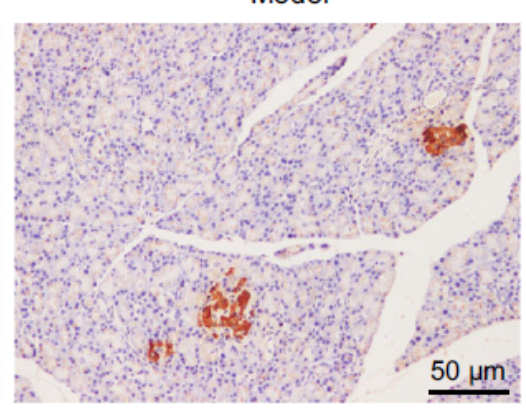

Figure 1

FBW7 is under-expressed in T1D mouse model. A) blood glucose level in the mouse model; B) Western blot results of FBW7 expression in islets from T1D mice $(n=10)$ and control mice $(n=10), *, p<0.05$, vs. normal group; $\mathrm{C}$ ) Representative images of $\mathrm{IHC}$ for FBW7 expression in islets from T1D mice and control mice $(\mathrm{bar}=50 \mu \mathrm{m})$. Measurement data are presented. Data comparisons between two groups were analyzed by unpaired t-test. Statistical analysis in relation to time-based measurements within each group was realized using repeated measures ANOVA. 

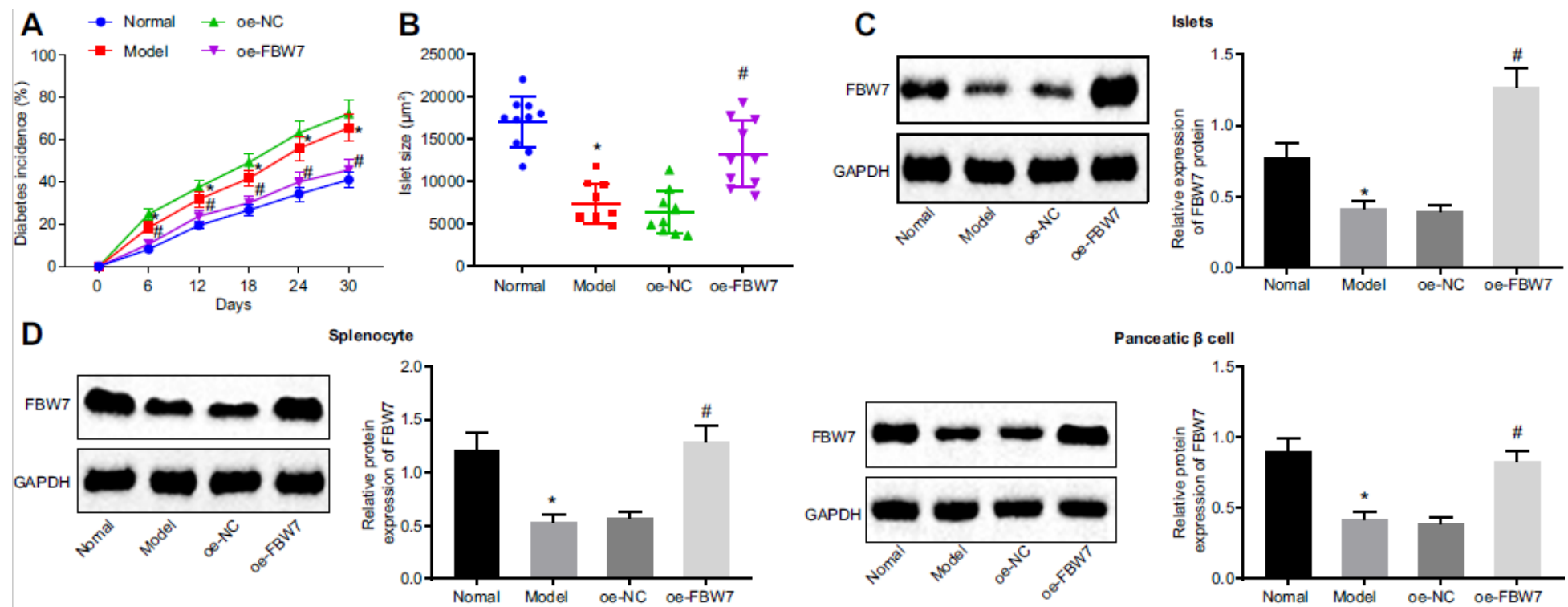

Panceatic $\beta$ cell

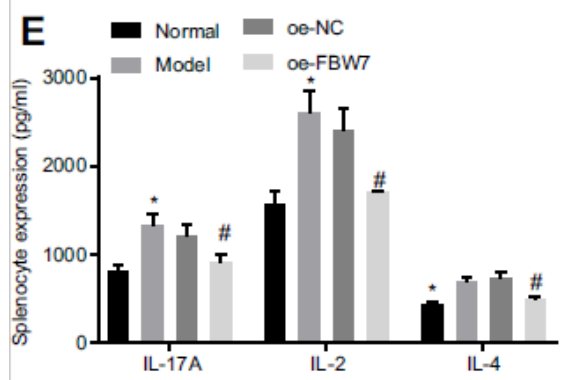

$\mathrm{F}$
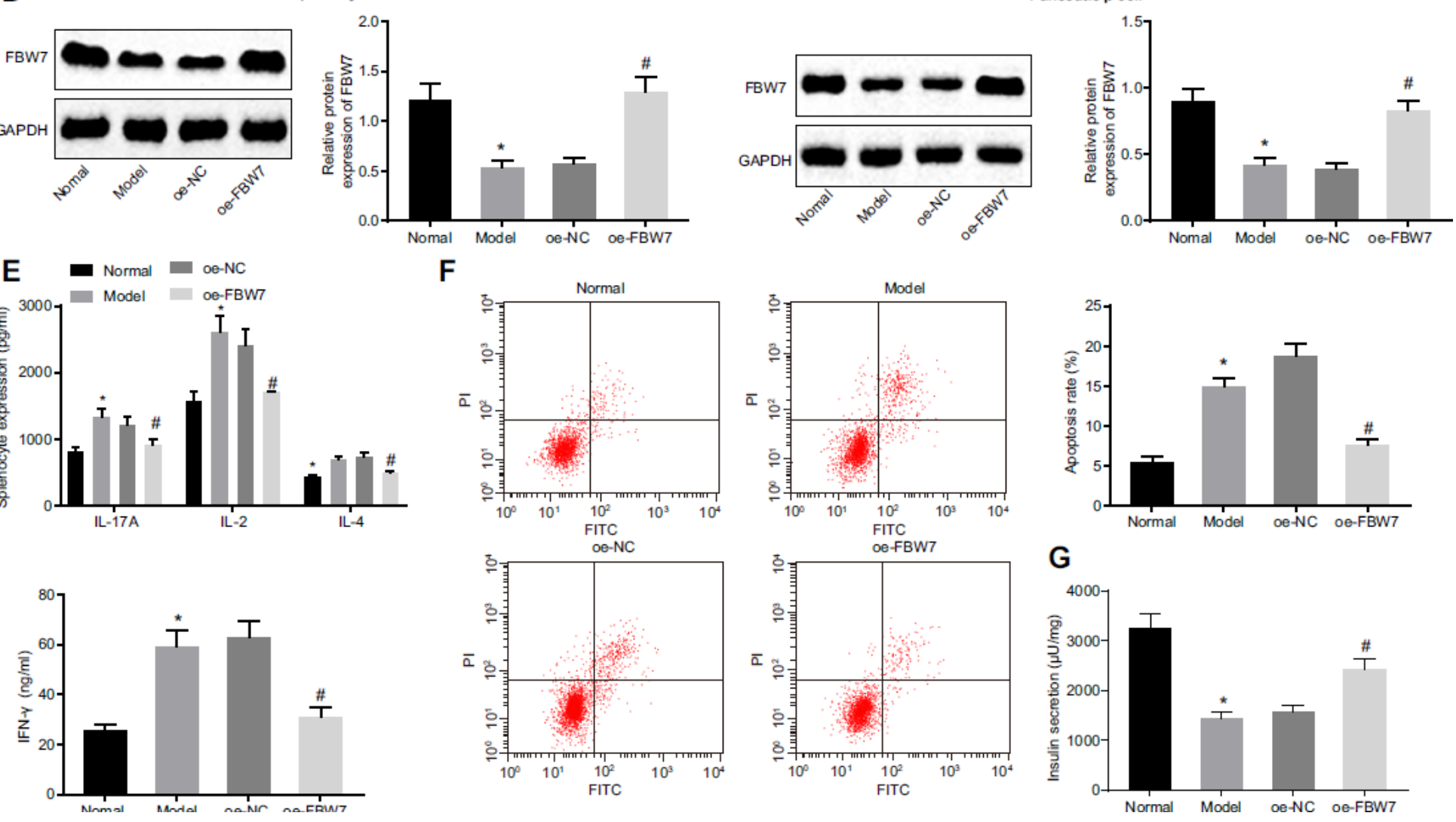

Figure 2

FBW7 decreases the occurrence of T1D in vivo and in vitro. A) diabetes incidence of each group, $*, p<$ 0.05 , vs. normal group, \#, $p<0.05$, vs. OE-NC group; B) islet size of each group, ${ }^{*}, p<0.05$, vs. normal group, \#, $p<0.05$, vs. OE-NC group; C) FBW7 expression in mice islet of each group determined by Western blot, *, $p<0.05$, vs. normal group, \#, $p<0.05$, vs. OE-NC group; D) FBW7 expression in induced splenocytes and pancreatic beta cells of each group determined by Western blot, $*, p<0.05$, vs. normal group, \#, $p<0.05$, vs. OE-NC group; E) level of IL-17AIIL-2IIFN-yIL-4 in cell supernatant of each group determined by ELISA, *, $p<0.05$, vs. normal group, \#, $p<0.05$, vs. OE-NC group; F) pancreatic beta cell apoptosis of each group determined by flow cytometry, ${ }^{*}, p<0.05$, vs. normal group, $\#, p<0.05$, vs. OE-NC group; G) insulin secretion of each group, *, $p<0.05$, vs. normal group, \#, $p<0.05$, vs. OE-NC group. All experiments were conducted in triplicate. Data comparisons between two groups were analyzed by unpaired t-test. Comparisons among multiple groups were performed by one-way ANOVA. Statistical analysis in relation to time-based measurements within each group was realized using repeated measures ANOVA. 
A

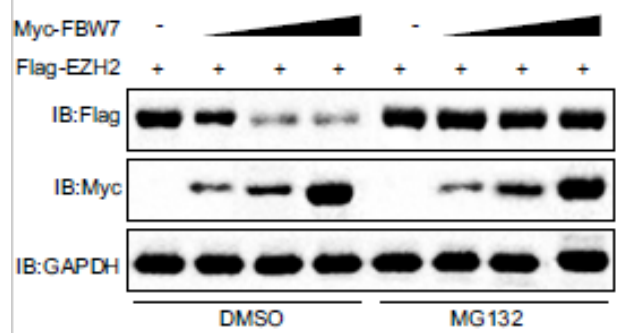

D
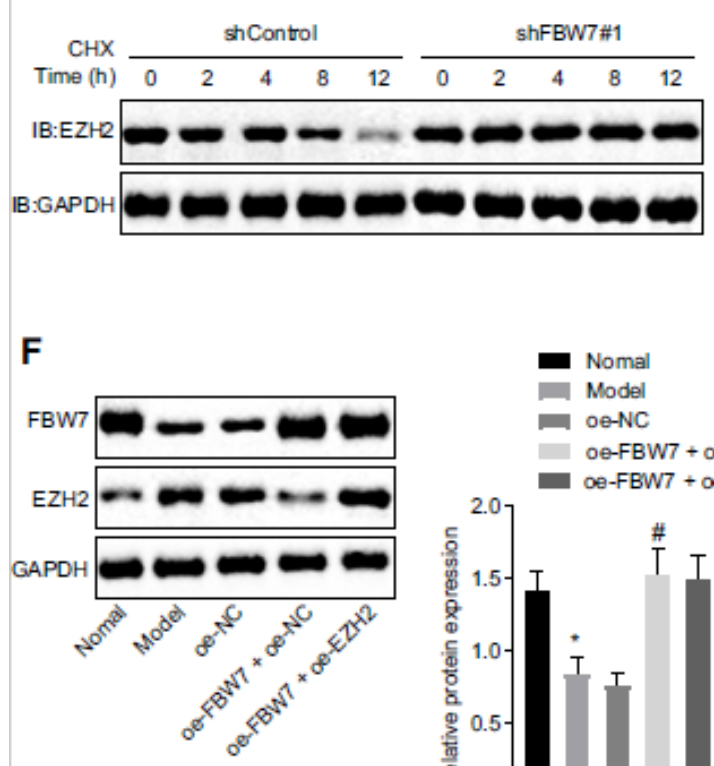

H

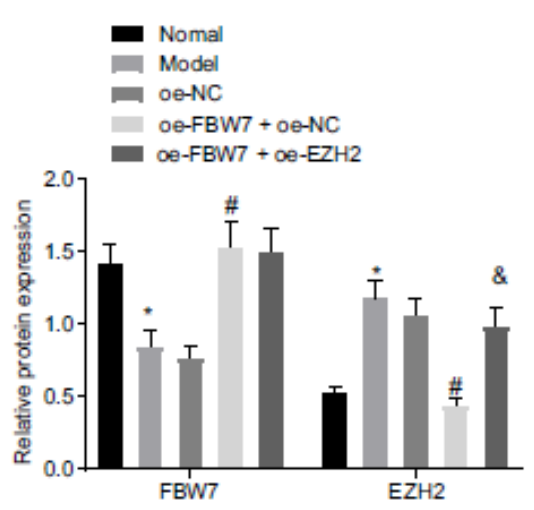

G

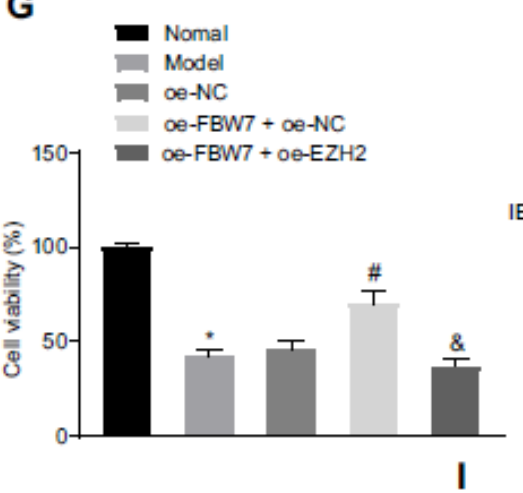

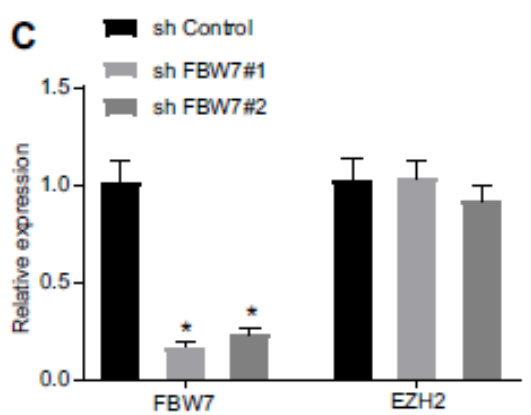

E

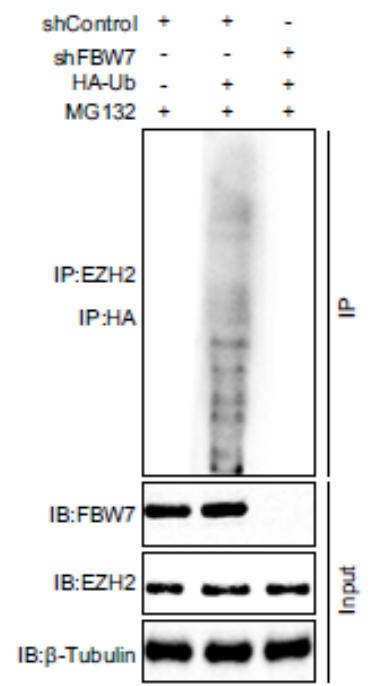

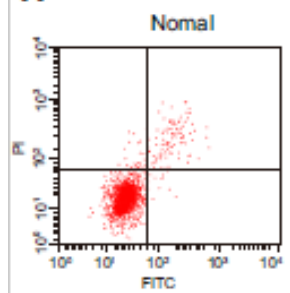
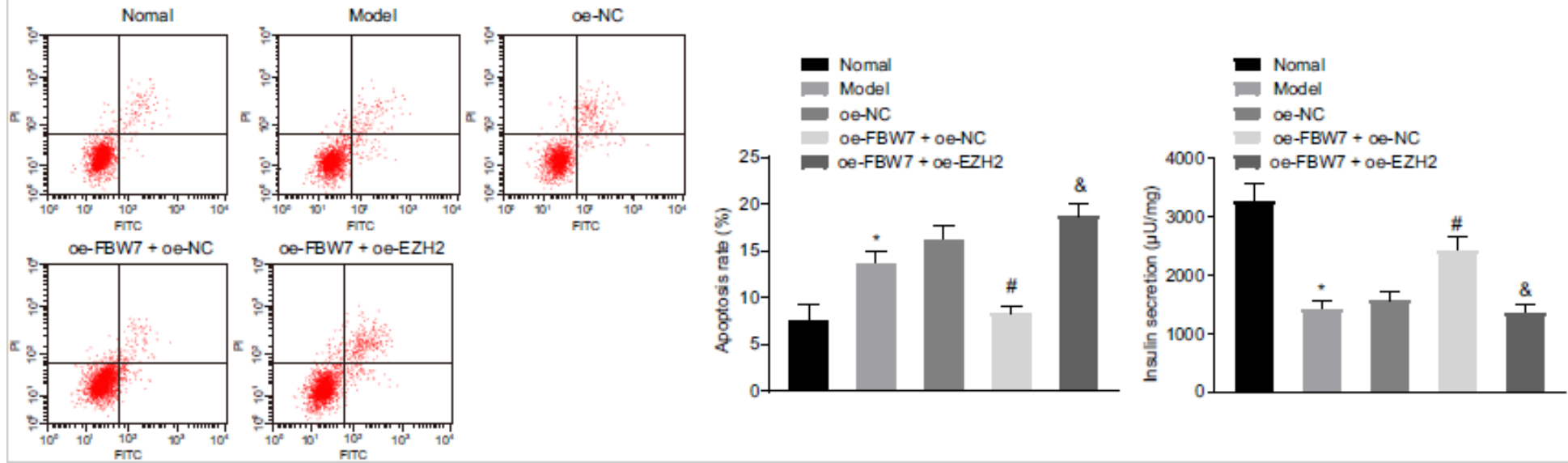

Figure 3

FBW7 inhibits TNF-a-induced pancreatic beta cells apoptosis and dysfunction through ubiquitin degradation of EZH2 A) Western blot assay of Min6 cells treated with MG132; B) Western blot assay of Min6 cells transfected with shFBW7, *, p < 0.05, vs. shControl group in FBW7 blotting, \#, p < 0.05, vs. shControl group in EZH2 blotting; C) RT-PCR quantification of FBW7 and EZH2 mRNA in Min6 cell transfected with shFBW7, *, p < 0.05, vs. shControl group; D) Western blot of EZH2 after CHX treatment at different time points, EZH2 was first normalized to GAPDH then normalized to time 0 at each time point, 
*, $p<0.05$, vs. shControl group; E) After 48 hrs of transfection of designed plasmid, the Min 6 cells was incubated with the MG132 $(20 \mu \mathrm{M})$ for $8 \mathrm{hrs}$, Western blot analysis of the EZH2 ubiquitination following with IP assay; F) Western blot assay of the FBW7 and EZH2 proteins in the FBW7-, or with EZH2-, overexpressed Min6 cells, *, $p<0.05$, vs. normal group, \#, $p<0.05$, vs. OE-EZH2 + OE-NC group, \&, $p<$ 0.05 , vs. OE-EZH2 + OE-FBW7 group; G) CCK-8 assay for cell viability in each group, *, $p<0.05$, vs. normal group, \#, p < 0.05, vs. OE-EZH2 + OE-NC group, \&, p< 0.05, vs. OE-EZH2 + OE-FBW7 group; H) flow cytometry determination for apoptosis in each group, *, p< 0.05, vs. normal group, $\#, p<0.05$, vs. OEEZH2 + OE-NC group, \&, p < 0.05, vs. OE-EZH2 + OE-FBW7 group; I) determination of insulin secretion, *, $p$ $<0.05$, vs. normal group, \#, p < 0.05, vs. OE-EZH2 + OE-NC group, $\&, p<0.05$, vs. OE-EZH2 + OE-FBW7 group. All experiments were conducted in triplicate. Data comparisons between two groups were analyzed by unpaired t-test. Comparisons among multiple groups were performed by one-way ANOVA. Statistical analysis in relation to time-based measurements within each group was realized using repeated measures ANOVA.

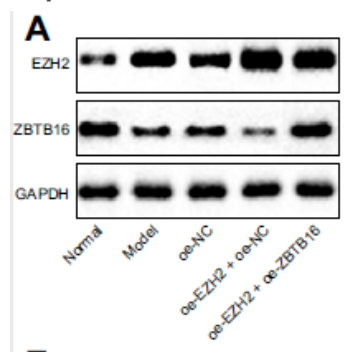

E
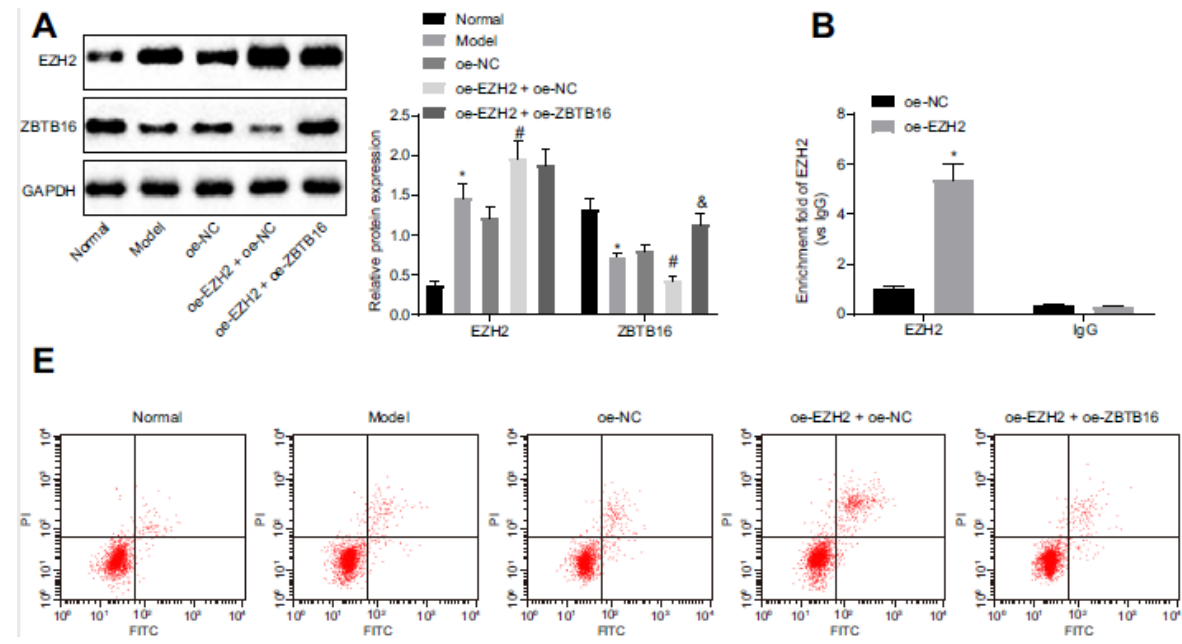
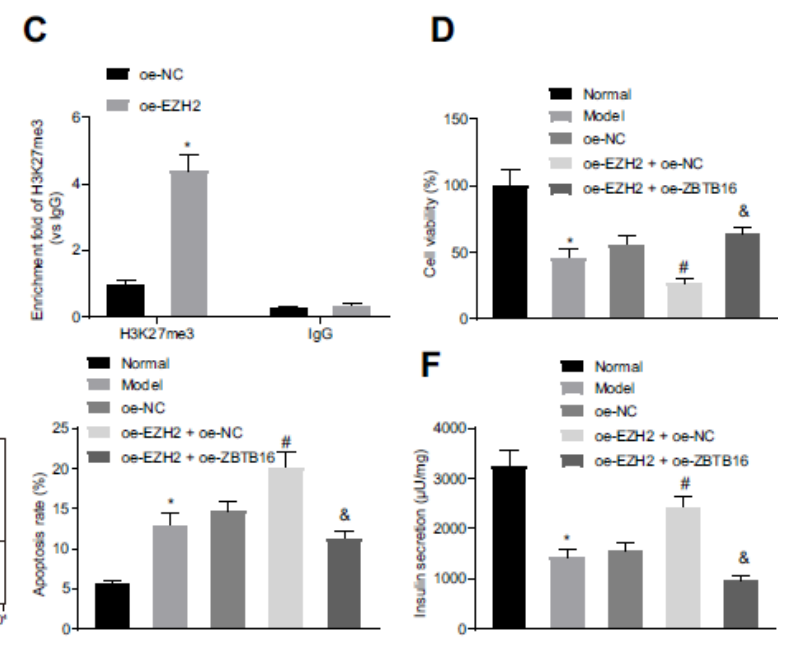

Figure 4

EZH2 enhances TNF-a-induced pancreatic beta cells apoptosis and dysfunction through ZBTB16 methylation A) Western blot assay of the EZH2 and ZBTB16 proteins in the EZH2-, or with ZBTB16-, overexpressed Min6 cells, *, $p<0.05$, vs. normal group, $\#, p<0.05$, vs. OE-EZH2 + OE-NC group, $\&, p<$ 0.05 , vs. OE-EZH2 + OE-ZBTB16 group; B) ChIP assay for detecting EZH2 accumulation in ZBTB16 promoter, *, $p<0.05$, vs. OE-NC group; D) ChIP assay for detecting H3K27me3 accumulation in ZBTB16 promoter, ${ }^{*}, p<0.05$, vs. OE-NC group; D) CCK-8 assay for cell variability, *, $p<0.05$, vs. normal group, \#, $p$ $<0.05$, vs. OE-EZH2 + OE-NC group, $\&, p<0.05$, vs. OE-EZH2 + OE-ZBTB16 group; E) flow cytometry assay for apoptosis, $*, p<0.05$, vs. normal group, \#, p< 0.05, vs. OE-EZH2 + OE-NC group, $\&, p<0.05$, vs. OEEZH2 + OE-ZBTB16 group; F) determination of insulin secretion, ${ }^{*}, p<0.05$, vs. normal group, $\#, p<0.05$, vs. OE-EZH2 + OE-NC group, \&, p < 0.05, vs. OE-EZH2 + OE-ZBTB16 group. All experiments were conducted in triplicate. Data comparisons between two groups were analyzed by unpaired t-test. Comparisons among multiple groups were performed by one-way ANOVA. 

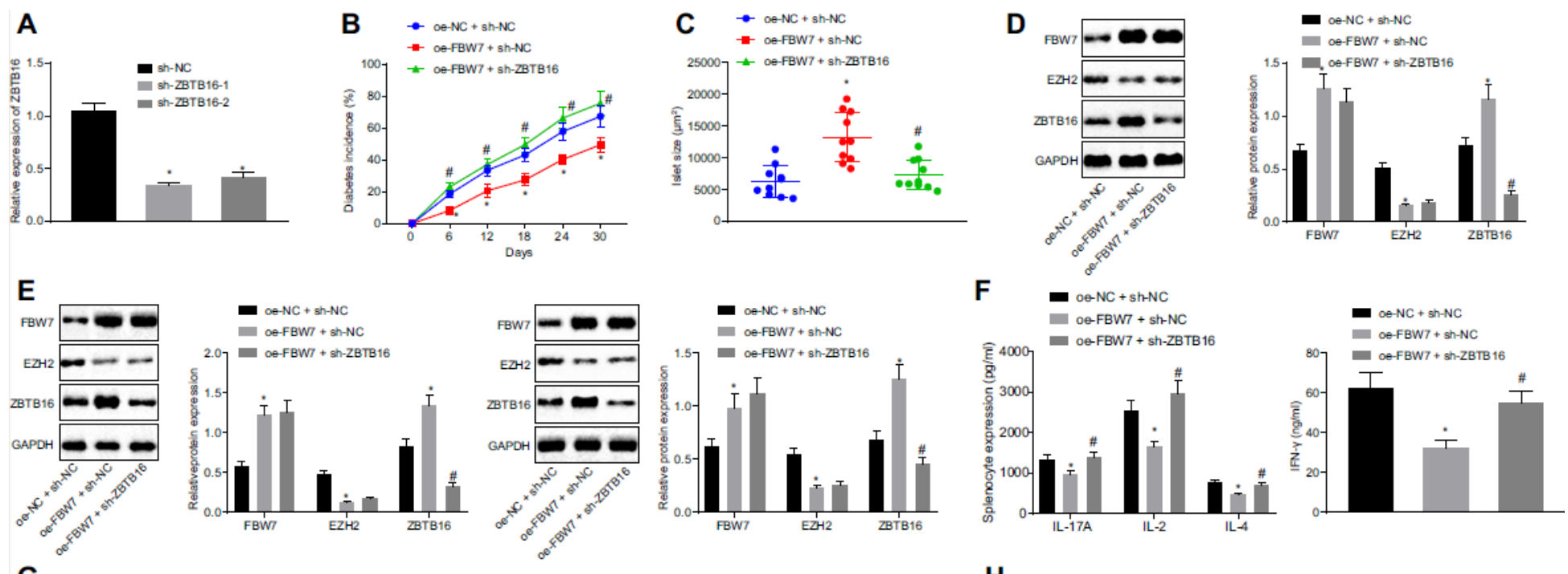

G
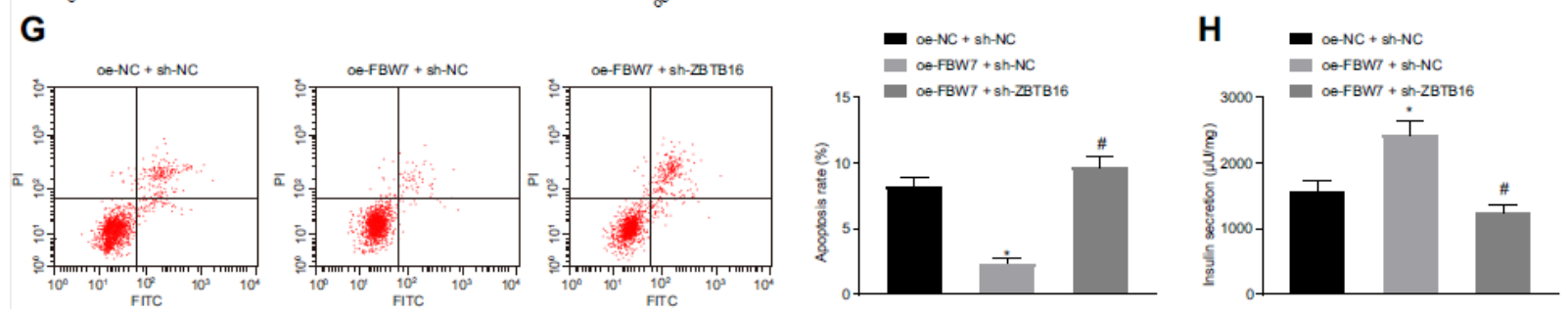

Figure 5

FBW7 inhibits T1D occurrence via EZH2/ZBTB16 in vivo and in vitro A) RT-PCR detection of ZBTB16 mRNA level in each group, ${ }^{*}, \mathrm{p}<0.05$, vs. OE-NC group; $\mathrm{B}$ ) diabetes incurrence in each mouse model group, *, $p<0.05$, vs. OE-FBW7 + sh-NC group; $C$ ) islet size in each mice model group, *, $p<0.05$, vs. OE-*, $p<0.05$, vs. OE-FBW7 + sh-NC group FBW7 + sh-NC group; D) Western blot detection of FBW7, EZH2, and ZBTB16 expression in islet, *, $p<0.05$, vs. OE-FBW7 + sh-NC group, \#, $p<0.05$, vs. OE-FBW7 + sh-NC group; E) Western blot detection of FBW7, EZH2, and ZBTB16 expression in splenocytes and pancreatic beta cells, *, $p<0.05$, vs. OE-FBW7 + sh-NC group, \#, $p<0.05$, vs. OE-FBW7 + sh-NC group; F) ELISA detection of IL-17AIIL-2 IIFN-y[IL-4 in cell supernatant, *, $p<0.05$, vs. OE-FBW7 + sh-NC group; G) flow cytometry detection of pancreatic beta cell apoptosis in each group, $*, p<0.05$, vs. OE-FBW7 + sh-NC group; $\mathrm{H}$ ) determination of insulin secretion, $*, p<0.05$, vs. OE-FBW7 + sh-NC group. All experiments were conducted in triplicate. Comparisons among multiple groups were performed by one-way ANOVA. Statistical analysis in relation to time-based measurements within each group was realized using repeated measures ANOVA. 


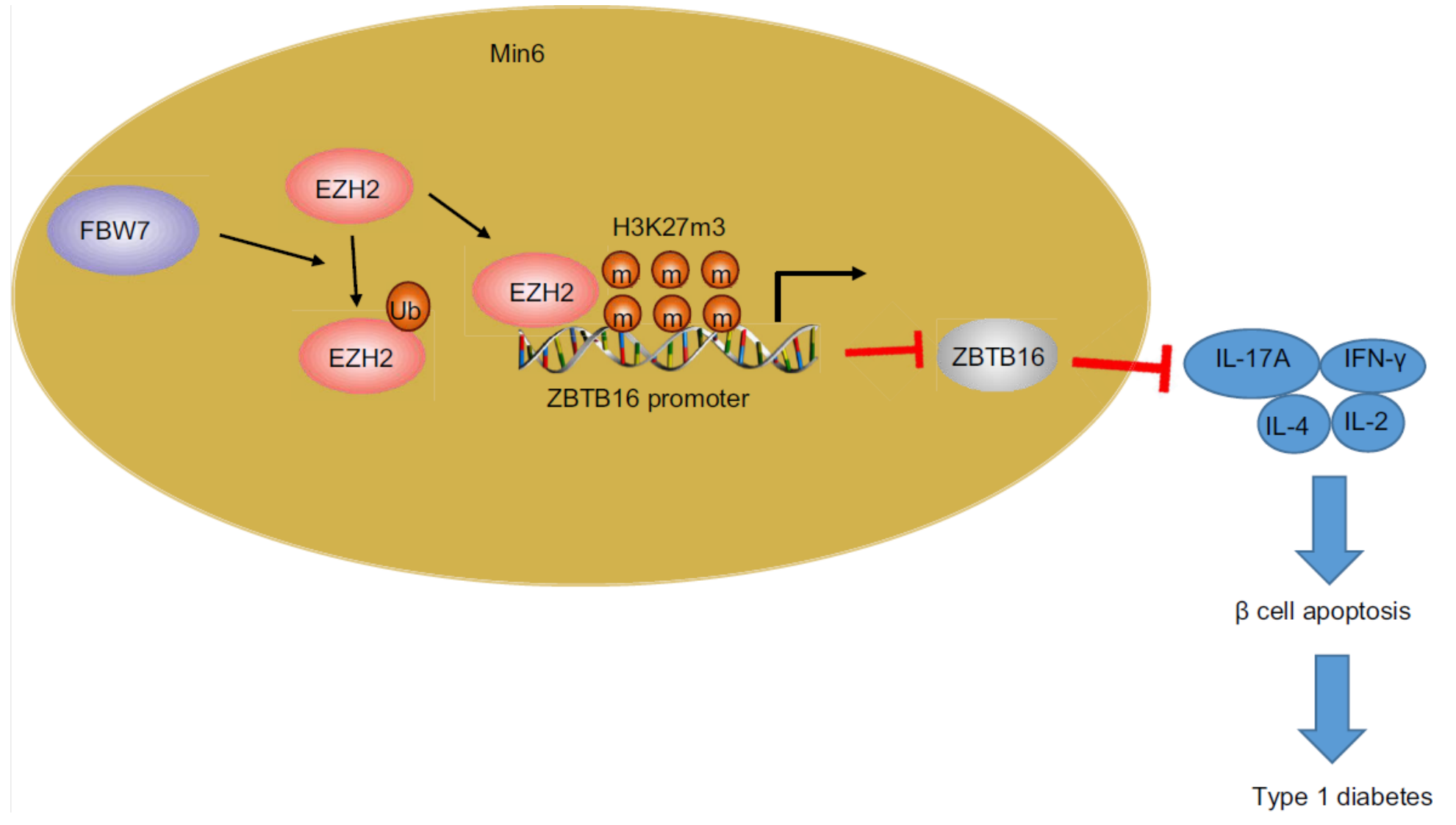

\section{Figure 6}

The mechanism graph of the regulatory network and function of FBW7. E3 ubiquitin ligase FBW7 prevents type I diabetes by upregulating ZBTB16 expression via histone methyltransferase EZH2. 